\section{European Venous Forum}

Der Besuch auf dem EVF Kongress in Zürich war nicht nur unglaublich „heiß“ von der Temperatur her - das Wetter schlug Hitze-Rekorde und der Glas-Kuppel-Saal für die Industrie war nicht klimatisiert - sondern auch vom Inhalt: Aus der ganzen Welt waren über 400 Abstracts für Vorträge eingegangen, davon wurden 30 selektiert und am Kongress vorgetragen, weitere 30 als Poster vorgestellt. Es handelt sich beim EVF um eine streng „Einsträngige“ Veranstaltung. Tatsächlich extrem interessante Forschungsergebnisse, innovativ und informativ! Ergänzt wurden die Abstracts-Sitzungen durch so genannte „didactic sessions“, in denen der Kongresspräsident, PD Dr. Dominik Heim aus Thun seine Kreativität einfließen ließ. Für mich persönlich war der Abschluss sehr erfreulich - in einer gemeinsamen Sitzung der EVF mit dem AVF (American Venous Forum) lag der Schwerpunkt des letzten Vortrags ganz im Sinne unserer Bemühungen, nicht alles zu therapieren, was machbar ist, sondern bei asymptomatischen Patienten möglichst einen Stammvenenerhalt anzustreben, insbesondere wenn die Stammvene nicht refluxiv ist (!).

Die Kandidatur der DGP, den EVF 2022 in Hamburg zu gestalten, wurde angenommen. Somit wird dieses internationale Treffen Ende Juni/Anfang Juli 2022 in Hamburg unter meiner Präsidentschaft (Erika Mendoza) stattfinden. Schon jetzt möchte ich alle forschenden Institutionen einladen, Studien vorzubereiten, die dort in Hamburg vorgestellt werden können. Besonders erfreulich war für mich die hohe Anzahl junger Forscher, die in Zürich referiert haben - dies wäre eine Ermunterung für unsere Universitäten. Jährlich werden auch zwei For- schungsprojekte ausgeschrieben und die besten mit 30.000 Euro Forschungszuschuss gefördert. Es wäre eine große Freude, wenn wir als Gastgeber es schaffen könnten, die deutsche phlebologische Forschung weiter voran zu bringen und uns präsentieren zu können. 